\title{
REPRESENTACIONES FAMILIARES SOBRE LA OBESIDAD INFANTIL EN DOS MUNICIPALIDADES DE LA HABANA
}

\author{
Leandro RODRÍGUEZ VÁZQUEZ; Maria Elena DÍAZ SÁNCHEZ \\ Instituto Nacional de Higiene, Epidemiología y Microbiología (Cuba) \\ leandrordguez@infomed.sld.cu, maryelen@infomed.sld.cu
}

\section{REPRESENTATIONS FAMILY ON CHILDHOOD OBESITY IN TWO MUNICIPALITIES OF HAVANA}

Resumen: Las representaciones alimentarias en la población constituyen un importante elemento para efectuar valoraciones acertadas sobre desórdenes alimentarios como el sobrepeso y la obesidad. Se realizo un estudio cualitativo a padres e hijos procedentes de escuelas de dos municipalidades de La Habana, Cuba. Se identificaron los niños sobrepeso y obesos por antropometría. Se aplicaron cuestionarios sobre estilos de vida y pruebas sobre la imagen corporal. Se realizaron grupos de discusión y encuestas a los padres. Se utilizó el método de la triangulación para analizar los resultados. El objetivo del trabajo es la identificación de las representaciones que posee la familia sobre los trastornos alimentarios con énfasis en la obesidad. Las discusiones grupales contribuyeron al acercamiento de esta complejidad que, por sus características requiere un enfoque multidisciplinario. Entre las manifestaciones más representativas se observó que existe problemas de autoestima y socialización, imagen corporal, no aceptación de la obesidad, poco conocimiento sobre la alimentación saludable y otros hábitos de vida beneficiosos. Se evidenció la poca percepción de riesgo de algunas familias sobre las consecuencias de la obesidad aunque cuando la identifican buscan ayuda profesional. La escuela y otros escenarios son adecuados para promover conductas saludables para disminuir el sobrepeso y la obesidad.

Abstract: Feeding representations are an important factor to make accurate evaluations of eating disorders such as overweight and obesity. A qualitative study of parents and schoolchildren in two municipalities of Havana, Cuba were done. The overweight and obese children were identified by anthropometry. Questionnaires on lifestyle and body image tests were applied. Discussion groups and surveys to the parents were conducted. Triangulation method was used to analyze the results. The study aims to identify the family representations about the eating disorders, with an emphasis on obesity. Discussion groups contributed to approach this complexity that, by their nature requires a multidisciplinary focus. Among the most representative manifestations were observed troubles of socialization and self-esteem, body image, lack in the acceptance of obesity, lack of knowledge about healthy eating and other beneficial life habits. The low perception of risk in some families about the consequences of obesity was evident even when it is the identified professional help is looked for. School and other settings are suitable to promote healthy behaviors to reduce overweight and obesity.

Palabras clave: Obesidad. Sobrepeso. Representaciones

Obesity. Overweight. Representations 


\section{Introducción}

Durante los últimos 15 años, el sobrepeso y la obesidad ha sufrido un notable incremento en Cuba (Díaz et al, 2014; Díaz et al, 2015)., que ha tenido repercusiones en la infancia (Jiménez, et al, 2013). Desde la perspectiva multidisciplinaria se pretende contribuir al pensamiento del fenómeno alimentario que representa el sobrepeso y la obesidad con una visión holística, educativa y cultural en la que se inserta el hombre y la mujer, desde lo cotidiano, que pudiera sugerir la existencia de una crisis en la alimentación de esas personas. Estas razones sustentan la necesidad de que la ciencia reclame de los estudiosos de la temática una dedicación especial para responder cuestionamientos y enfoques epistemológicos que no permitan que se lacere a la cultura alimentaria sin valorar sus componentes. Más allá de las estadísticas que implican una generalización de los fenómenos, se debe considerar lo que experimentan los sujetos que sufren el sobrepeso y la obesidad a través de los enfoques cualitativos.

Desde este mismo enfoque, la cultura como fenómeno alimentario requiere vislumbrarse por los conocimientos, las tradiciones, las creencias, los valores, la identidad y las representaciones o estereotipos, entre otros, para activar el conjunto de las actividades que se establecen por los grupos humanos y obtener del entorno los alimentos que posibilitan su subsistencia. Estos últimos abarcan desde la producción, la distribución, el almacenamiento, la conservación y la preparación hasta su consumo, e incluyen todos los aspectos simbólicos que acompañan las diferentes fases del proceso. La cultura alimentaria se erige como el sustento de la convergencia de las diversas disciplinas que abordan la alimentación en el contexto biocultural.

El abordaje de las representaciones en el desarrollo de la educación y la cultura alimentarias son aspectos complejos que requieren de un análisis desprejuiciado. En este sentido, la identificación de las prácticas, los hábitos, las costumbres, así como el simbolismo corporal brindan el acerbo de conocimientos que son importantes para la prevención ulterior del sobrepeso y la obesidad, lo cual requiere del apoyo de los actores sociales en las comunidades e intervenir en ellas.

El objetivo del trabajo se centra en la identificación de las representaciones que posee la familia sobre el sobrepeso y la obesidad.

\section{Materiales y Métodos}

Se realizó un estudio cualitativo en familias de estudiantes sobrepeso y obesos de quinto y sexto grado, en dos escuelas primarias de diferentes municipalidades de La Habana, Cuba. La muestra se concibió a criterio, a partir de todos los estudiantes con sobrepeso y obesos evaluados por antropometría. Los padres fueron citados y asistieron a grupos de discusión, con posterioridad fueron visitados en los hogares. Inicialmente se aplicó un cuestionario ${ }^{1}$ a los estudiantes sobre hábitos de vida que incluyó la diversidad alimentaria para constatar las opiniones de los niños y las niñas con las de sus familiares en grupos de discusión y entrevistas.

En la primera escuela se citaron 29 familias de las niñas y los niños. Asistieron un total de 10 familias a un grupo de discusión en presencia de maestros y facilitadores; estuvieron presentes 7 madres, 2 abuelas y 1 abuelo. En la segunda escuela asistieron 16 familiares al grupo de discusión: 13 madres, 1 abuela, 2 padres y educadores. Se identificaron como informantes a los padres, abuelas y abuelos, así como al personal docente. Se realizó una

1 Cuestionario NIÑOS, elaborado y validado por el Departamento de Tecnología de la Alimentación y Nutrición. Universidad Católica San Antonio de Murcia (UCAM). 
entrevista a profundidad en el hogar a las familias para identificar criterios acerca del sobrepeso y la obesidad, personales y de sus hijos, en su relación con la alimentación saludable.

Se evaluó la imagen corporal de los niños en la escuela y de los padres durante las entrevistas; adicionalmente a estos último se les pidió valorar la imagen corporal de sus hijos; se utilizaron instrumentos estandarizados para las evaluaciones. (Osuna-Ramírez et al 2006; Harris el al, 2008).

El análisis de los datos se efectuó a través de la triangulación de todas las fuentes de información: cuestionario, grupos de discusión, entrevista semiestructurada grabada, notas de campo, documentos científicos teóricos y experimentales. Previamente se siguió el proceso de recolección de datos en formato de audio y video, trascripción, codificación abierta, y confección de matrices para la reducción de la información, que permitieron la comprensión. La categorización fue desarrollada sobre los supuestos: imagen corporal, relaciones sociales, herencia, actividad física, economía familiar, formas de alimentación y riesgo para la salud.

\section{Resultados}

En el cuestionario sobre hábitos de vida aplicado a todos los escolares, se pudo constatar que sobre la diversidad alimentaria en su mayoría los niños gustan de los dulces, las chucherías, las pastas con énfasis en las pizzas y los alimentos fritos. En cuanto a las frutas y vegetales existen distinciones en las representaciones de las dos escuelas, pudiéndose constatar diferencias en el conocimiento de su valor desde el punto de vista nutricional. En la primera se observó que los niños y niñas poseen ideas escasas acerca de la importancia de las frutas y los vegetales para la alimentación saludable. La segunda escuela reveló resultados que apuntan a una mayor comprensión sobre su significado nutritivo.

Del análisis de los grupos de discusión y las entrevistas a partir de los informantes se pudo constatar que en la relación alimentación/obesidad/salud los padres coinciden en que el sobrepeso y la obesidad:

1. Repercute en los cánones de belleza y la estética.

2. Se afecta la autoestima al manifestar que sus hijos se sienten inconformes con sus cuerpos.

3. Es determinante el papel de la herencia (muchos apuntan a que en sus familias hay personas obesas o "corpulentas").

4. Ven a la obesidad como un problema que recién descubren.

5. Los niños y niñas de $6^{\text {to }}$ grado, al pasar a la enseñanza secundaria son los que más sufren de diferentes maneras y se acentúa en la adolescencia, entre otras razones.

6. La situación económica puede rivalizar con la percepción que otros estudiantes manifiestan sobre sus cuerpos y favorece la discriminación.

7. La constitución física para diferenciar a las criollitas o envueltas en carne de las gordas u obesas constituye una representación de la idiosincrasia en la sociedad cubana.

8. El consumo excesivo de algunos medicamentos puede ser un factor desencadenante.

9. La actividad física es insuficiente y poco rigurosa.

10. Los niños y niñas realizan poca actividad desde la educación física y el ejercicio dedicado al esparcimiento y los deportes; ven mucha televisión, dedican muchas horas al uso de la computadora como distracción. Los juegos que realizaban los niños de otras generaciones, se han olvidado.

11. El exceso de alimentos puede aportar energía abundante que se refleja en el cuerpo de las personas.

12. Las personas pican alimentos entre las comidas. 
13. Se dificulta la gestión de los alimentos debido a situaciones económicas y escases de tiempo.

14. Los padres se sienten culpables, porque no asumen la responsabilidad que se tiene ante este aspecto.

15. Ser obesos es estar enfermo y tener otras complicaciones

\section{Discusión}

Las representaciones alimentarias son parte de la cultura que influye en los comportamientos relacionados con el sobrepeso y la obesidad. Comprender el fenómeno alimentario desde el individuo y la familia, es considerar de manera holística algunos de los factores que intervienen en el proceso. Esta perspectiva, considera a los alimentos como parte de la dieta y la cultura en las dimensiones históricas, biológicas, económicas, políticas y sociales. La pedagogía, psicología y filosofía; pudieran favorecer estos análisis de una manera más integral.

El desarrollo de la cultura de la alimentación saludable se puede fomentar a través del establecimiento de vínculos con el desarrollo de la educación alimentaria, precisamente porque desde los inicios de la humanidad, el hombre comenzó a transmitir los conocimientos que iba incorporando (Uda et al, 2005). De ahí la importancia de que en este proceso, una generación transmita a las siguientes el legado de armonía y salud en el plano individual, familiar y comunitario en sus relaciones sociales, que repercuten en el desarrollo de la obesidad como entidad compleja.

Disimiles preguntas se formularon en los diferentes escenarios, sobre la obesidad y sus consecuencias: Las madres confundieron la valoración de la obesidad al asociarla como gordura, relacionada con el peso de la persona, que influye en la belleza corporal y otras concepciones estéticas vinculadas con la moda, en el niño y la niña, así como en el adulto. Creen que es una expresión física o forma corporal, que a menudo puede estar representada en todos o en algunos integrantes de la familia y que en sus hijos les afectan de forma negativa, tanto la autoestima como la condición física en la actualidad y en el futuro inmediato. Llamó a la reflexión el hecho de que los padres consideran,

que la sociedad a veces es cruel con las personas obesas, se utilizan expresiones para identificarlas, como gordita, gordito y aunque no es lo común, el maestro que con cariño y amor dice: la gordita de mi grupo puede cantar, el gordito puede hacer esto o aquello, aunque también es una forma de distinguirlos, al ser diferentes a lo que se supone que sea la media, indiscutiblemente, en estas ingenuas frases se esta cometiendo maltrato.

Lo anterior lacera la integridad y por supuesto, repercute desfavorablemente en la salud mental y fisiológica de estos individuos. Otros criterios sobre la percepción de los padres acerca del sobrepeso y la obesidad de sus hijos o hijas destacan que no les agrada verlos en ese estado, porque se sienten incómodos y no les gusta. Algunos refieren que:

no se les nota mucho, que es sólo la barriguita y que nunca han mencionado bajar de peso". Sin embargo, reconocen que "se acomplejan por la forma en que los demás los tratan, porque en esas edades comienzan a presumir, se acercan a los estudios secundarios y piensan que deben estar bonitos.

Para otros progenitores el exceso de peso de sus hijos transcurre de forma temporal y llegará a desaparecer con el tiempo en la adolescencia, pero si persistiera podría constituirse en un problema de índole estético, que trae como consecuencia dificultades de socialización 
con sus pares. En síntesis, la mayoría de los familiares posee una conceptualización de la gordura diferente a la caracterización de la obesidad; lo primero asociado con un exceso de masa corporal y lo segundo como una enfermedad que puede generar otras complicaciones como la diabetes e hipertensión y las asociadas al corazón.

En los grupos de discusión desarrollados impacta a la familia el desconocimiento que poseen acerca del estado nutricional de sus hijos. Describen que han sido gorditos desde pequeños y que no consideraban que eso fuera un problema de obesidad. Esto denota que el término obesidad la identifican más en su relación con la salud que las palabras gorditos y gorditas, debido a que en las creencias populares, la gordura es sinónimo de buena salud.

Aunque los padres plantearon que muchos de sus hijos sufren más por la situación económica y cómo van vestidos a la escuela (aunque se use uniforme)., que por el sobrepeso y la obesidad. En realidad, aunque ellos no aceptan el problema, este fenómeno debe ser abordado, porque impacta en la psiquis de los muchachos y ese nivel de estrés genera desequilibrios que provocan ansiedad por la comida, sin importar el alimento que se ingiere. En otras situaciones sí se define la obesidad como un problema, que los padres niegan en sus hijos y no tienen noción de sus implicaciones, lo que provoca que su proceso de individualización se retarde. Resultados semejantes se reportan en niños y niñas de otros países (Tognarelli, 2012; Núñez Rivas et al, 2013).

Los padres mostraron preocupación debido a que existen medicamentos que provocan obesidad porque aceleran el apetito e inducen al exceso de peso. Es evidente que algunos fármacos alteran el mecanismo fisiológico que controlan el estado nutricional y por ende el peso corporal; este mecanismo está determinado por el balance que existe entre el aporte calórico y el gasto energético de cada individuo. Algunos de los medicamentos que se utilizan por ejemplo, en psiquiatría para mejorar la función conductual del individuo, provocan un aumento del apetito y otros efectos secundarios como la somnolencia que favorecen una actitud sedentaria. Los antihistamínicos y esteroides son otros que estimulan esta condición. Por lo tanto, habría que vigilar la alimentación y el peso con estos tipos de tratamientos debido a que pueden provocar sobrepeso y obesidad.

El retardo en la aparición del exceso de peso en estos individuos pudiera abordarse con la modificación de los estilos de vida. Estas acciones han sido recomendadas sobre todo en las enfermedades multifactoriales y en las terapias de la obesidad (Coyote-Estrada y MirandaLora, 2008). En estas últimas, siempre que impliquen la utilización de medicamentos, es necesario controlar la aparición del sobrepeso secundario, el tiempo y la duración del tratamiento.

En opinión de los padres, la disminución de los juegos al aire libre y actividades dinámicas como las que se desarrollan en la escuela, acompañados de horas frente a la televisión, los videojuegos y las computadoras son ingredientes para el desencadenamiento del sobrepeso y la obesidad, lo cual ha sido reportado en otros escenarios científicos y académicos (Faith et at, 2001; Amigo Vázquez, et al, 2008).

En las discusiones grupales surgieron criterios sobre la duración de la educación física en las escuelas, que deben incrementarse en la frecuencia, con ciclos variados y por cada año escolar practicar varios deportes. Además de planificarse otras actividades físicas atendiendo a las áreas con que cuenten los centros educacionales y la atención individualizada a los niños y niñas con exceso de peso.

En la actualidad la familia no participa en las actividades lúdicas de sus hijos, ni en otras que pueda beneficiar la calidad de vida de todos. Existen opciones de juegos tradicionales, estos no son frente a la computadora. La complejidad se visualiza como un problema que no es ajeno al contexto cubano ya que muchos países han identificado estas situaciones (Álvarez y Díaz, 1996; Benítez, 2000). Los padres deben asumir protagonismo, al incorporar actividades que provoquen el incremento del gasto energético para contribuir a la disminución del sobrepeso y la obesidad. 
Este fenómeno no es un problema local, y pasa también por determinadas condiciones materiales y complejidades de los núcleos familiares. Las niñas más que los niños de la generación actual están mucho más tiempo huyéndole a la ejercitación física en cualquier escenario, sea en la casa o en la escuela, entre semanas o en los fines de semanas. No se trata de luchar contra la modernidad, sino incrementar la actividad física de los niños y niñas en su relación con la familia.

A criterio de los padres se vive en tiempos acelerados, lo que condiciona que no se pueda estar mucho con los hijos y esta situación favorece que el niño se estrese y la ansiedad cause el deseo de comer y como consecuencia, aumente de peso. Se producen afectaciones, como se ha referido, dejarlos deliberadamente entretenidos frente a la televisión durante horas; en estas circunstancias comer cualquier cosa frente a ésta, en la mayoría de las ocasiones estiran la mano y saben lo que comen por el sabor y el olor de los alimentos. Esta inactividad puede conducir, acompañada de malos hábitos de alimentación, al sobrepeso y la obesidad.

Ante la pregunta de ¿Qué es la obesidad? se constataron opiniones que la vinculan, fundamentalmente, con la cantidad de comida que se ingiere, así como con los modos de prepararlas y de comerlas. Entre los alimentos que se identifican como responsables de esta problemática se destacan: las comidas y la pica a base de alimentos energéticos, elaborados esencialmente con harinas refinadas, los que contienen cantidades de grasa por encima de lo recomendado y las chucherías (caramelos, bombones, etc.).

En el imaginario social la representación de las frutas y los vegetales como alimentos de interés en la comidas de los pequeños, se afecta debido a que diversas familias no consideran entre sus prácticas la incorporación de estos, por varias razones, entre las que se pueden mencionar, los precios que dificultan el acceso y el desconocimiento de sus beneficios para la salud.

Otro aspecto se refiere a las interrogantes sobre las preferencias alimentarias de los hijos:

La familia cubana de estos tiempos no le pregunta al niño lo que desea comer porque hay que ajustarse a lo que exista en los mercados y al dinero que se disponga, y si resulta incómodo para los mayores, ¿cómo sucederá para los pequeños? Se priorizan a los niños con lo les gusta sin preguntarles, porque no es una práctica habitual. Tampoco se acostumbra a reunirse en familia durante los horarios de desayuno, almuerzo y comidas.

En el contexto de las discusiones grupales se destacó que por regla general, algunos fines de semana socializan el acto de comer, pero no representa una costumbre. Estas regularidades se presentan de acuerdo a la situación socioeconómica de la familia.

En este sentido, cocinar en familia no es una práctica sistemática de los grupos estudiados. Ciertos niños les gusta ayudar en la elaboración de las comidas, pero generalmente los adultos alegan estar apurados y no se dispone del tiempo necesario para que los hijos participen. No obstante, algunos destacan el interés por estudiar carreras afines con la cocina o ser cocineros. En otro sentido, "dejarles que participen los hace independientes, por lo que hay que enseñarles a freír un huevo, dejarles hacer algunas tareas de la casa, fregar su plato si es el único que come en ese momento y además, que preparen la leche”. Aunque no es una experiencia que se manifiesta en todas las familias, este tipo de actividad permite socializar las prácticas culinarias y las costumbres alimentarias y trasmitirlas de una generación a otra. De manera que es también una forma de asimilar y conservar la cultura de los pueblos.

El conflicto se produce cuando lo que se cocina no es lo que a ellos les gusta a los niños. Una madre refiere:

En el caso de los míos son machos, yo soy la reina, esclava de la cocina, trato de complacer a todos en la semana pero cuando pongo la comida en la mesa 
no hay opción, se trata de balancear los gustos de todos". Otra informante expresa que "cuando no les gusta lo que se prepara para el almuerzo o la comida de la tarde, sencillamente, no lo comen y se acuestan sin comer, puede que se coman un pan y no les gusten, rechazan todo lo demás y no hay quien los obligue en ese sentido. Cuando dicen no, es no.

En las discusiones de grupo y las entrevistas a profundidad se destacó que:

los horarios de las comidas, no siempre es posible satisfacerlos. En la escuela se cumple el horario de almuerzo, pero después, pasan muchas horas sin comer nada hasta que llegan a la casa, esto les causa una necesidad de comer cualquier cosa y toman una merienda. En el caso contrario, salen a la calle y compran lo que se les ocurre.

Otros enfatizan el interés de los niños por los horarios y el servicio en la mesa de los alimentos, pues en la asignatura de Educación Laboral se les enseña desde quinto grado, como poner la mesa y elaborar algunos alimentos. En sentido general, la escuela contribuye a que se adopten prácticas alimentarias saludables. No obstante, cuando el niño permanece en ella desde el almuerzo hasta pasadas las cuatro y treinta de la tarde, salen a la calle a buscar algo de comer. Entonces se favorece la desestructuración alimentaria, debido posiblemente, a que con el consentimiento de los padres hacen lo que no deben y favorecer el sobrepeso y la obesidad.

En ocasiones los hijos les acompañan a realizar la compra de alimentos. Generalmente, no se les da participación en la adquisición de éstos en los agromercados. Son los padres los que deciden lo más saludable, por tanto lo que compran, otras veces se les complace si desean algo en específico. Los padres deben aprender a escuchar los criterios de sus hijos, por lo que, una buena práctica sería, instruirles para que aprendan desde pequeños a reconocer los alimentos más saludables y el comportamiento de la economía familiar. En sentido general, los adultos consideran que sus hijos, solo quieren caramelos, refrescos, chocolates, sobre todo lo que se vende en algunos establecimientos comerciales.

Por regla general, la familia cubana no suele comer en la calle, en la actualidad es esporádico debido al precio que tienen los alimentos en los restaurantes, lo cual no significa que no se efectúe, pero cuando sucede, los hijos deciden qué comer de acuerdo al presupuesto y priorizan que sus pequeños se sientan complacidos y satisfechos.

En otro orden, se pudo constatar que los padres coinciden, en que la cultura se hereda con deformaciones en materia de alimentación, la dieta básica del cubano no es precisamente para este clima; es una dieta que se apoya en leguminosas, arroz, disimiles alimentos fritos y no existe un balance entre lo que se oferta y lo que se demanda por parte de la población. En las prácticas culinarias existe monotonía y por tanto, se convierten en un patrón que se reproduce.

El fenómeno es extremadamente complejo, de manera que desde la familia primero, la escuela (Freire, 2009). y los medios masivos de difusión deben ser portadores de mensajes útiles para que cada individuo, desde las edades tempranas comience a establecer el cuidado por la alimentación saludable, precisamente, porque ella garantizará que las demás funciones del organismo sean adecuadas (Figueroa Pedraza, 2003). y esto contribuye a la lucha contra sobrepeso y la obesidad.

Se pudo constatar que cuando los padres reconocieron que sus hijos eran sobrepesos y obesos, trataron de buscar la orientación adecuada. Otros los incorporaron a las actividades deportivas con restricciones de algunos alimentos, sin seguir criterios médicos que avalaran la planificación de la alimentación. Las mediciones antropométricas y las discusiones en los debates grupales permitieron la sensibilidad de la familia ante esta compleja situación. 
Alternativamente, algunos familiares poseen claridad con respecto a que la primera que puede influir en esta situación es la familia, pero es necesario que consulten a un especialista en nutrición para que sea efectiva la ayuda, sobre todo porque en ocasiones los niños no comprenden las limitaciones impuestas de dejar de comer determinados alimentos hipercalóricos.

Al considerar las expectativas sobre los debates focales y grupales, los padres coincidieron en que estos fueron productivos, que los hijos deben participar en ellos aunque se participen de otras actividades en la escuela. De esta manera que se establece un compromiso entre padres e hijos para modificar o realizar acciones para disminuir los efectos de uso y abuso de la ingestión inadecuada de alimentos y evitar el sobrepeso y la obesidad.

Lo anterior refuerza la necesidad de que la familia desempeña un papel de primer orden, de modo que la complejidad alimentaria debe ser discutida con ella, porque se involucra la participación activa de los hombres y las mujeres para propiciar el cambio de conductas en beneficio de la salud. En estas discusiones la mujer se reafirma como la hacedora de la cultura familiar, empieza a forjarse una nueva percepción de sí misma y las relaciones estructurales de este conglomerado humano asignarán nuevos retos. En las condiciones actuales se requiere de la mujer una nueva cultura, insertada en el ecosistema, para poder pensar y actuar como eje de la familia y la comunidad con una visión ambientalista-ecosófica, y con una fuerte base económico-familiar que se dirija a la obtención de alimentos que contribuyan al logro de la alimentación saludable. La obesidad por su parte ya es un problema mundial que tiene que se inserta el contexto histórico reciente, en el que se involucra lo económico con repercusiones sociales, el desarrollo de la ciencia y la tecnología y las prácticas culturales de los individuos en su hábitat.

\section{Comentarios finales}

La interpretación de las representaciones encontradas acerca del sobrepeso y la obesidad reafirma que la familia, en su mayoría las madres, señalan cómo el niño o la niña con sobrepeso y obesidad se acompleja por lo estético y no están conformes con su cuerpo, tiene problemas de socialización con sus pares y pueden desarrollar enfermedades en la edad adulta. Confunden el término de gordura con obesidad y no tienen percepción de la alimentación adecuada y su repercusión en la salud. El sedentarismo lo asocian con el aumento de peso corporal, no obstante, no accionan para disminuir sus consecuencias. Cuando finalmente detectan el problema en sus hijos es que toman conciencia y buscan la ayuda profesional.

Ofrecer actividades orientadas desde la escuela y otros escenarios para los niños, niñas y familias, así como el personal docente aun cuando el curso escolar concluya es importante. El personal salubrista y los líderes comunitarios pudieran ofrecer acciones de promoción de salud en beneficio de la alimentación saludable con énfasis en la disminución del sobrepeso y la obesidad.

\section{Agradecimientos}

Los autores agradecen a las autoridades escolares, padres, alumnos y otros informantes por su participación desinteresada en el estudio. De igual modo a todos los miembros del equipo de investigación por contribución en el desarrollo de este trabajo.

\section{Bibliografía}

Álvarez, M. Díaz M. (1996). La familia cubana. Situación actual y proposiciones para su fortalecimiento. CIPS. Serie de Monografía UNICEF/CUBA. No. 14. La Habana

Amigo Vázquez I, Busto Zapico R, Herrero Díez J y Fernández Rodríguez C. (2008). Actividad física, ocio sedentario, falta de sueño y sobrepeso infantil. Psicothema 20 (4).: 516-520 
Benítez, M.E. (2000). Panorama sociodemográfico de la familia cubana. Editorial Ciencias Sociales, La Habana.

Coyote-Estrada N, Miranda-Lora A L. (2008). Tratamiento farmacológico de la obesidad en niños. Bol Medico Hospital Infantil México 65: 547-567

Diaz M E, Garcia Roche R. (2014). Nuevos datos del sobrepeso y obesidad adulta en Cuba. Nutición clínica y dietética hospitalaria 34, supl. 1 (7).: 153.

Díaz ME, García Roche R, Jiménez S, Bonet M. (2015). Tendencias en el estado nutricional de la población cubana entre 1995 y 2010. ANTHROPOS. XII Simposio de Antropología Física "Luis Montané": La Antropología ente los desafíos del siglo XXI. La Habana. Research Gate DOI: 10.13140/RG.2.1.1338.5764.

Faith M S, Berman N, Heo M, Pietrobelli A, Gallagher D, Epstein L H, Eiden M T, Allison DB. (2001). Effects of Contingent Television on Physical Activity and Television Viewing in Obese Children. Pediatrics 107: 1043-1049.

Figueroa Pedraza, D. (2003). Seguridad alimentaria familiar. Revista. Salud pública y nutrición Mexico.; 4 (2).: http://www.respyn.uanl.mx/index.html. (17-10- 2006).

Freire, P. (2009). Pedagogía del oprimido. Ed. Caminos. La Habana.

Harris CV, Bradlyn AS, Coffman J, Grunel E, Cottrell L. (2008). BMI-based body size guides for women and men: development and validation of a novel pictorial method to assess weightrelated concepts. International Journal of Obesity 32: 336-342.

Jiménez Acosta SM, Rodríguez Suárez A, Díaz Sánchez ME. La obesidad en Cuba. (2013). Una mirada a su evolución en Diferentes grupos poblacionales. Revista Cubana de Alimentación y Nutrición 23 (2).: 297-308.

Núñez Rivas HP, Campos Saborio N, Alfaro Mora FV, Holst Schumacher I. (2012). Las creencias sobre obesidad de niños y niñas en edad Escolar y las de sus progenitores. Rev. Actualidades Investigativas en Educación 13 (2).: 1-30.

Osuna-Ramírez I, Hernández-Prado B, Campuzano JC, Salmerón J. (2006). Índice de masa corporal y percepción de la imagen corporal en una población adulta mexicana: la precisión del autorreporte. Salud Publica México 48 :94-103.

Tognarelli Guzmán A. (2012). Representaciones de apego de niños y niñas con obesidad y la respuesta sensible de sus madres. Summa Psicológica Ust, Vol. 9, № 2, 57-67.

Uda A N, Díaz de Aliaga, M, Tomas, M. C. (2005). Educación Alimentaria Nutricional (E.A.N.). Escuela de Nutrición de la Facultad de Ciencias Médicas de la Universidad Nacional de Córdoba.

\section{$\cos$}

